PROCEEDINGS OF THE

AMERICAN MATHEMATICAL SOCIETY

Volume 131, Number 11, Pages 3535-3546

S 0002-9939(03)06960-0

Article electronically published on March 25, 2003

\title{
SUPERCYCLIC AND CHAOTIC TRANSLATION SEMIGROUPS
}

\author{
MAI MATSUI, MINO YAMADA, AND FUKIKO TAKEO
}

(Communicated by Joseph A. Ball)

\begin{abstract}
We give a necessary and sufficient condition for a translation semigroup to be supercyclic and to be chaotic in a weighted function space.
\end{abstract}

\section{INTRODUCTION}

For a strongly continuous semigroup $\{T(t)\}$ on a Banach space $X$, W. Desch, W. Schappacher and G. F. Webb 1] investigated conditions for a semigroup to be hypercyclic or chaotic. They gave the spectral properties for a semigroup to be chaotic and gave a necessary and sufficient condition to be hypercyclic for a translation semigroup on a weighted function space $L_{\rho}^{p}(I)$ or $C_{0, \rho}(I)$ by using the property of an admissible weight function. A semigroup is called hypercyclic if there exists $x \in X$ such that $\{T(t) x \mid t \geq 0\}$ is dense in $X$ and supercyclic if there exists $x \in X$ such that $\{c T(t) x \mid t \geq 0, c \in \mathbf{R}\}$ is dense in $X$. The hypercyclic semigroup is said to be chaotic if in addition the set of periodic points is dense in $X$. D. A. Herrero et al. investigated the spectral properties of hypercyclic and supercyclic operators on a complex, separable infinite-dimensional Hilbert space [2, 3].

In this paper we investigate the property of admissible weight functions which induce supercyclic, hypercyclic and chaotic translation semigroups on a weighted function space $L_{\rho}^{p}(I)$ or $C_{0, \rho}(I)$. As for supercyclicity, a strongly continuous semigroup on $L_{\rho}^{p}(I)$ or $C_{0, \rho}(I)$ is always supercyclic if $I$ is an interval $[0, \infty$ ) (Theorem 11)). For $I=(-\infty, \infty)$, the semigroup is not always supercyclic and we give a necessary and sufficient condition to be supercyclic for the translation semigroup on a weighted function space $L_{\rho}^{p}(I)$ or $C_{0, \rho}(I)$ (Theorem $\mathbb{1}(2)$ ). We also construct a special function $x$ such that $\{c T(t) x \mid t \geq 0, c \in \mathbf{R}\}$ is dense in $X$ (see the remark following the proof of Theorem 1 ). Although the necessary and sufficient condition for the semigroup to be hypercyclic and supercyclic does not depend on $L_{\rho}^{p}(I)$ or $C_{0, \rho}(I)$ but depends on the interval $I=[0, \infty)$ or $I=(-\infty, \infty)$, the condition to be chaotic depends on $L_{\rho}^{p}(I)$ or $C_{0, \rho}(I)$. We give a necessary and sufficient condition for the semigroup to be chaotic (Theorems 2 and 3), and show the relation between the condition and $\lim _{\tau \rightarrow \infty} \rho(\tau)=0$ (Examples 1 and 2). We show a necessary and sufficient condition for $\rho$ to satisfy $\lim _{\tau \rightarrow \infty} \rho(\tau)=0$, which implies more than that $X_{\text {per }}$ (the set of periodic points of $X$ ) is dense in $X$ (Theorem 4 ).

Received by the editors December 7, 2001 and, in revised form, June 21, 2002.

2000 Mathematics Subject Classification. Primary 47D60; Secondary 47D06.

Key words and phrases. Admissible weight function, supercylic semigroups, chaotic semigroups. 


\section{Preliminaries}

Let $X$ be a real Banach space. A strongly continuous semigroup $\{T(t)\}$ of linear operators on $X$ is called supercyclic (resp. hypercyclic) if there exists $x \in X$ such that $\{c T(t) x \mid t \geq 0, c \in \mathbf{R}\}$ (resp. $\{T(t) x \mid t \geq 0\}$ ) is dense in $X$ [3]. A strongly continuous semigroup $\{T(t)\}$ is called chaotic if $\{T(t)\}$ is hypercyclic and the set $X_{\text {per }}=\{x \in X \mid \exists t>0$ s.t. $T(t) x=x\}$ of periodic points is dense in $X$ [1].

Let $I$ be the interval $[0, \infty)$ or $(-\infty, \infty)$. By an admissible weight function on $I$ we mean a measurable function $\rho: I \rightarrow \mathbf{R}$ satisfying the conditions:

(i) $\rho(x)>0$ for all $x \in I$;

(ii) there exist constants $M \geq 1$ and $\omega \in \mathbf{R}$ such that $\rho(x) \leq M e^{\omega t} \rho(t+x)$ for all $x \in I$ and $t>0$.

With an admissible weight function, we construct the following function spaces:

$$
\begin{aligned}
L_{\rho}^{p}(I)= & \left\{u: I \rightarrow \mathbf{R} \mid u \text { measurable, } \int_{I}|u(\tau)|^{p} \rho(\tau) d \tau<\infty\right\} \\
& \text { with }\|u\|=\left(\int_{I}|u(\tau)|^{p} \rho(\tau) d \tau\right)^{\frac{1}{p}} \quad(p \geq 1), \\
C_{0, \rho}(I)= & \left\{u: I \rightarrow \mathbf{R} \mid u \text { continuous, } \lim _{\tau \rightarrow \pm \infty} \rho(\tau) u(\tau)=0\right\} \\
& \text { with }\|u\|=\sup _{\tau \in I}|u(\tau)| \rho(\tau) .
\end{aligned}
$$

We consider a (forward) translation semigroup $\{T(t)\}$ with parameter $t \geq 0$ such as

$$
[T(t) u](\tau)=u(\tau+t) \quad \text { for } u \in C_{0, \rho}(I) \text { or } L_{\rho}^{p}(I) .
$$

When $\rho(\tau)=1$, weighted function spaces are equal to $L^{p}(I)$ or $C_{0}(I)$ and the translation semigroup is never hypercyclic, since the norm of $T(t)$ is equal to 1 for all $t \geq 0$ in $L^{p}(I)$ or $C_{0}(I)$. A necessary and sufficient condition for the translation semigroup in $L_{\rho}^{p}(I)$ or $C_{0, \rho}(I)$ to be hypercyclic is known as follows.

Theorem A (1). Let $X$ be $L_{\rho}^{p}(I)$ or $C_{0, \rho}(I)$ with an admissible weight function $\rho$. Then the following (1) and (2) are equivalent:

(1) the translation semigroup $\{T(t)\}$ on $X$ is hypercyclic;

(2) (i) if $I=[0, \infty)$, then $\liminf _{t \rightarrow \infty} \rho(t)=0$ holds,

(ii) if $I=(-\infty, \infty)$, then for each $\theta \in \mathbf{R}$ there exists a sequence $\left\{t_{j}\right\}_{j=1}^{\infty}$ $\left(t_{j} \rightarrow \infty\right.$ as $\left.j \rightarrow \infty\right)$ of positive real numbers such that $\lim _{j \rightarrow \infty} \rho\left(t_{j}+\theta\right)=$ $\lim _{j \rightarrow \infty} \rho\left(-t_{j}+\theta\right)=0$.

As for the property of an admissible function of $\rho$ there is the following lemma.

Lemma B (1). Let I be the interval $(-\infty, \infty)$ or $[0, \infty)$ and let $\rho$ be an admissible weight function on $I$, that is, there exists $M \geq 1$ and $\omega \in \mathbf{R}$ such that $\rho(\tau) \leq$ $M e^{\omega t} \rho(\tau+t)$ for all $\tau \in I$ and $t>0$. For $l>0$, put $M_{l}=M e^{\omega l}$ for $\omega>0$ and $M_{l}=M$ for $\omega \leq 0$. Then $M_{l} \geq 1$ and the inequality

$$
\frac{1}{M_{l}} \rho(\sigma) \leq \rho(\tau) \leq M_{l} \rho(\sigma+l)
$$

holds for any $\sigma \in I$ and any $\tau \in[\sigma, \sigma+l]$. 


\section{Supercyclic translation semigroups on $L_{\rho}^{p}(I)$ and $C_{0, \rho}(I)$}

As shown in the previous section, a necessary and sufficient condition for the translation semigroup to be hypercyclic is known. In this section, we shall give necessary and sufficient conditions for the translation semigroup to be supercyclic on $L_{\rho}^{p}(I)$ and $C_{0, \rho}(I)$.

Lemma 1. Let $\{T(t)\}$ be a strongly are equi continuous semigroup on a Banach space $X$, such that for some $x \in X,\{c T(t) x \mid t \geq 0, c \in \mathbf{R}\}$ is dense in $X$. Then the following assertions hold:

(1) $T(t) x \neq 0$ for all $t \geq 0$;

(2) the set $\{c T(t) x \mid t \geq s, c \in \mathbf{R}\}$ is dense in $X$ for all $s \geq 0$.

Proof. (1) Suppose that $t_{0}>0$ is minimal with the property that $T\left(t_{0}\right) x=0$. We show first that each $y \in X$ is of the form $y=c T(t) x$ for some $t \in\left[0, t_{0}\right]$ and $c \in \mathbf{R}$. There is a sequence $t_{n} \in\left[0, t_{0}\right]$ and a sequence $c_{n} \in \mathbf{R}$ such that $c_{n} T\left(t_{n}\right) x \rightarrow y$. Without loss of generality we may assume that $t_{n}$ converges to some $t$. Assume first that $t=t_{0}$. In this case we have

$$
\begin{aligned}
& \left\|T\left(t_{0}-t_{n}\right) y\right\|=\left\|T\left(t_{0}-t_{n}\right)\left(y-c_{n} T\left(t_{n}\right) x\right)+c_{n} T\left(t_{0}\right) x\right\| \\
& \quad \leq\left\|T\left(t_{0}-t_{n}\right)\right\|\left\|y-c_{n} T\left(t_{n}\right) x\right\| \rightarrow 0 .
\end{aligned}
$$

Since $T\left(t_{0}-t_{n}\right) y \rightarrow y$ we obtain the trivial case $y=0$. On the other hand, if $t<t_{0}$, then $\left\|T\left(t_{n}\right) x\right\|$ is bounded away from 0 . By compactness we may assume that $c_{n}$ converges to some $c$ and we infer that $y=c T(t) x$.

Now take three vectors $y_{i}=c_{i} T\left(t_{i}\right) x \in X$, spanning a two-dimensional subspace, such that each pair $y_{i}, y_{j}, i \neq j$, is linearly independent. Assume that $t_{1}>t_{2}>t_{3}$. We have then $y_{3}=\alpha_{1} y_{1}+\alpha_{2} y_{2}$. Now we arrive at the contradiction

$$
\begin{aligned}
0 & \neq c_{3} T\left(t_{0}+t_{3}-t_{2}\right) x=T\left(t_{0}-t_{2}\right) y_{3}=\alpha_{1} T\left(t_{0}-t_{2}\right) y_{1}+\alpha_{2} T\left(t_{0}-t_{2}\right) y_{2} \\
& =\alpha_{1} c_{1} T\left(t_{0}+t_{1}-t_{2}\right) x+\alpha_{2} c_{2} T\left(t_{0}\right) x=0 .
\end{aligned}
$$

(2) Suppose that there exists $s_{0} \geq 0$ such that $A=\left\{c T(t) x \mid t \geq s_{0}, c \in \mathbf{R}\right\}$ is not dense in $X$. Hence there exists a bounded open set $U$ such that $U \cap \bar{A}=\varnothing$. Therefore we have

$$
U \subset \overline{\left\{c T(t) x \mid 0 \leq t \leq s_{0}, c \in \mathbf{R}\right\}}
$$

by using the relation

$$
X=\overline{\{c T(t) x \mid t \geq 0, c \in \mathbf{R}\}}=\overline{\left\{c T(t) x \mid t \geq s_{0}, c \in \mathbf{R}\right\}} \cup \overline{\left\{c T(t) \mid 0 \leq t \leq s_{0}, c \in \mathbf{R}\right\}} .
$$

Since $T(t) x$ is continuous with $t$ and $T(t) x \neq 0$ holds for all $t \geq 0$ by Lemma 11), there exists $m_{1}, m_{2} \in \mathbf{R}$ such that $0<m_{1} \leq\|T(t) x\| \leq m_{2}$ for $0 \leq t \leq s_{0}$. There exists $M \geq 0$ such that $\|y\| \leq M$ for any $y \in U$ because $U$ is bounded. So we have $U \subset \overline{\left\{c T(t) x\left|0 \leq t \leq s_{0},\right| c \mid \leq \frac{M}{m_{1}}\right\}}$, which means that $\bar{U}$ is compact. Hence $X$ is finite dimensional, which contradicts that $X$ is infinite dimensional.

Lemma 2. Let $\{T(t)\}$ be a strongly continuous semigroup on a Banach space $X$. Then the following are equivalent:

(1) $\{T(t)\}$ is supercyclic;

(2) for all $y, z \in X$ and all $\varepsilon>0$, there exists $v \in X, t>0$ and $c \in \mathbf{R}$ such that $\|y-v\|<\varepsilon$ and $\|z-c T(t) v\|<\varepsilon ;$ 
(3) for all $y, z \in X$, all $\varepsilon>0$ and for all $l \geq 0$, there exists $v \in X, t>l$ and $c \in \mathbf{R}$ such that $\|y-v\|<\varepsilon$ and $\|z-c T(t) v\|<\varepsilon$.

Proof. (1) implies (3): Let $\{c T(t) x \mid t \geq 0, c \in \mathbf{R}\}$ be dense in $X$. For any $y, z \in X$ and any $l \geq 0$, there exists $s>0$ and $c_{1} \in \mathbf{R}$ such that $\left\|y-c_{1} T(s) x\right\|<\varepsilon$, and there exists $u>s+l$ and $c_{2} \in \mathbf{R}$ such that $\left\|z-c_{2} T(u) x\right\|<\varepsilon$ by Lemma 2. Put $v=c_{1} T(s) x$. Then we have the first inequality. Put $t=u-s>l$ and $c=\frac{c_{2}}{c_{1}}$. Then we have the second inequality.

(3) implies (2): It is obvious.

(2) implies (1): The proof is similar to the proof in the case of hypercyclic in [1.

Using these lemmas, we give a necessary and sufficient condition for a translation semigroup to be supercyclic.

Theorem 1. Let $X$ be the space $L_{\rho}^{p}(I)$ or $C_{0, \rho}(I)$ and $\rho$ be an admissible weight function. Let $\{T(t)\}$ be a strongly continuous semigroup on $X$. Then the following assertions hold:

(1) if $I=[0, \infty)$, then $\{T(t)\}$ is supercyclic;

(2) if $I=(-\infty, \infty)$ and $\{T(t)\}$ is a translaion semigroup, then $\{T(t)\}$ is supercyclic if and only if for any $\theta \in \mathbf{R}$ there exists a sequence $\left\{t_{j}\right\}_{j=1}^{\infty}\left(t_{j} \rightarrow \infty\right.$ as $j \rightarrow \infty)$ such that $\lim _{j \rightarrow \infty} \rho\left(t_{j}+\theta\right) \rho\left(-t_{j}+\theta\right)=0$.

Proof. (1) Let $X_{0}$ be the set of all $x \in X$ such that the support of $x$ is compact. For any $y, z \in X$ and any $\varepsilon>0$, there exists $y_{0} \in X_{0}$ such that $\left\|y-y_{0}\right\|<\frac{\varepsilon}{2}$ since $X_{0}$ is dense in $X$. There exists $t_{1}>0$ such that $T(s) y_{0}=0$ for any $s \geq t_{1}$ since $y_{0} \in X_{0}$. Put

$$
\omega^{\prime}(\tau)= \begin{cases}z\left(\tau-t_{1}\right) & t_{1} \leq \tau \\ \frac{z(0)}{\varepsilon} \tau+z(0)\left(1-\frac{t_{1}}{\varepsilon}\right) & t_{1}-\varepsilon \leq \tau \leq t_{1}, \\ 0 & 0 \leq \tau \leq t_{1}-\varepsilon .\end{cases}
$$

Then $T\left(t_{1}\right) \omega^{\prime}=z$ holds. Put $\omega=\frac{\varepsilon \omega^{\prime}}{2\left\|\omega^{\prime}\right\|}, c=\frac{2\left\|\omega^{\prime}\right\|}{\varepsilon}$ and $v=y_{0}+\omega$. Then we have $v \in X,\|y-v\|=\left\|y-y_{0}-\omega\right\| \leq\left\|y-y_{0}\right\|+\|\omega\|<\varepsilon$ and $\left\|z-c T\left(t_{1}\right) v\right\| \leq$ $\left\|z-c T\left(t_{1}\right) \omega\right\|+\left\|c T\left(t_{1}\right) \omega-c T\left(t_{1}\right) v\right\|=\left\|z-T\left(t_{1}\right) \omega^{\prime}\right\|+\left\|c T\left(t_{1}\right) y_{0}\right\|=0$. By Lemma $22),\{T(t)\}$ is supercyclic.

(2) We shall show the proof in the case $X=L_{\rho}^{p}(I)(p \geq 1)$.

$(\Rightarrow)$ Let $\{T(t)\}$ be supercyclic. We will show that $\lim _{j \rightarrow \infty} \rho\left(t_{j}+\theta\right) \rho\left(-t_{j}+\theta\right)=0$.

Fix any $\theta \in \mathbf{R}$. Let $y, z \in X$ be functions with compact support $\subset[\theta, \theta+l] \quad(l>$ 0 ), $y \geq 0, z \leq 0$, and $\|y\|=\|z\|=1$. By Lemma 2[3), for any $\varepsilon>0$ there exists $v_{\varepsilon} \in X, t_{\varepsilon}>l$ and $c_{\varepsilon}>0$ such that $\left\|c_{\varepsilon} T\left(t_{\varepsilon}\right) v_{\varepsilon}-z\right\|<\varepsilon$ and $\left\|v_{\varepsilon}-y\right\|<\varepsilon$. Put $\omega_{1}=v_{\varepsilon \mid[\theta, \theta+l]}^{+}$and $\omega_{2}=v_{\varepsilon \mid\left[\theta+t_{\varepsilon}, \theta+l+t_{\varepsilon}\right]}^{-}$.

Then we have the following: $\omega_{1} \geq 0, \operatorname{supp}\left(\omega_{1}\right) \subset[\theta, \theta+l], \operatorname{supp}\left(T\left(t_{\varepsilon}\right) \omega_{1}\right) \subset$ $\left[\theta-t_{\varepsilon}, \theta+l-t_{\varepsilon}\right], \omega_{2} \leq 0, \operatorname{supp}\left(\omega_{2}\right) \subset\left[\theta+t_{\varepsilon}, \theta+l+t_{\varepsilon}\right]$ and $\operatorname{supp}\left(T\left(t_{\varepsilon}\right) \omega_{2}\right) \subset[\theta, \theta+l]$. Then the following hold:

$$
\begin{aligned}
\left\|c_{\varepsilon} T\left(t_{\varepsilon}\right) \omega_{1}\right\| & <\varepsilon, \\
\|y\|-\left\|\omega_{1}\right\| & <\varepsilon, \\
\left\|\omega_{2}\right\| & <\varepsilon, \\
\|z\|-\left\|c_{\varepsilon} T\left(t_{\varepsilon}\right) \omega_{2}\right\| & <\varepsilon .
\end{aligned}
$$


By Lemma B, there exists $M_{l} \geq 1$ satisfying (1). Then the following hold:

$$
\begin{aligned}
&\left\|c_{\varepsilon} T\left(t_{\varepsilon}\right) \omega_{1}\right\|^{p}=\int_{\theta-t_{\varepsilon}}^{\theta+l-t_{\varepsilon}} \rho(\tau)\left|c_{\varepsilon} \omega_{1}\left(\tau+t_{\varepsilon}\right)\right|^{p} d \tau \\
& \geq \frac{1}{M_{l}} \rho\left(\theta-t_{\varepsilon}\right)\left|c_{\varepsilon}\right|^{p} \int_{\theta-t_{\varepsilon}}^{\theta+l-t_{\varepsilon}}\left|\omega_{1}\left(\tau+t_{\varepsilon}\right)\right|^{p} d \tau \\
&=\frac{\left|c_{\varepsilon}\right|^{p}}{M_{l}} \rho\left(\theta-t_{\varepsilon}\right) \int_{\theta}^{\theta+l}\left|\omega_{1}(\tau)\right|^{p} d \tau \\
&\left\|\omega_{1}\right\|^{p}=\int_{\theta}^{\theta+l} \rho(\tau)\left|\omega_{1}(\tau)\right|^{p} d \tau \leq M_{l} \rho(\theta+l) \int_{\theta}^{\theta+l}\left|\omega_{1}(\tau)\right|^{p} d \tau .
\end{aligned}
$$

So we have the inequality

$$
\frac{\left\|\omega_{1}\right\|^{p}}{M_{l} \rho(\theta+l)} \leq \frac{M_{l}\left\|c_{\varepsilon} T\left(t_{\varepsilon}\right) \omega_{1}\right\|^{p}}{\left|c_{\varepsilon}\right|^{p} \rho\left(\theta-t_{\varepsilon}\right)}
$$

Similarly we have the following:

$$
\begin{gathered}
\left\|\omega_{2}\right\|^{p}=\int_{\theta+t_{\varepsilon}}^{\theta+l+t_{\varepsilon}} \rho(\tau)\left|\omega_{2}(\tau)\right|^{p} d \tau \geq \frac{1}{M_{l}} \rho\left(\theta+t_{\varepsilon}\right) \int_{\theta+t_{\varepsilon}}^{\theta+l+t_{\varepsilon}}\left|\omega_{2}(\tau)\right|^{p} d \tau, \\
\left\|c_{\varepsilon} T\left(t_{\varepsilon}\right) \omega_{2}\right\|^{p}=\int_{\theta}^{\theta+l} \rho(\tau)\left|c_{\varepsilon} \omega_{2}\left(\tau+t_{\varepsilon}\right)\right|^{p} d \tau \leq M_{l} \rho(\theta+l)\left|c_{\varepsilon}\right|^{p} \int_{\theta+t_{\varepsilon}}^{\theta+l+t_{\varepsilon}}\left|\omega_{2}(\tau)\right|^{p} d \tau .
\end{gathered}
$$

So we have the inequality

$$
\frac{M_{l}|| \omega_{2} \|^{p}}{\rho\left(\theta+t_{\varepsilon}\right)} \geq \frac{\left\|c_{\varepsilon} T\left(t_{\varepsilon}\right) \omega_{2}\right\|^{p}}{M_{l}\left|c_{\varepsilon}\right|^{p} \rho(\theta+l)}
$$

By the inequalities (3.1a), (3.2a), and (3.1b),

$$
\begin{aligned}
\varepsilon^{p} & >\left\|c_{\varepsilon} T\left(t_{\varepsilon}\right) \omega_{1}\right\|^{p} \geq \frac{\left.\left|c_{\varepsilon}\right|^{p} \rho\left(\theta-t_{\varepsilon}\right)|| \omega_{1}\right|^{p}}{M_{l}^{2} \rho(\theta+l)} \\
& >\frac{\left|c_{\varepsilon}\right|^{p} \rho\left(\theta-t_{\varepsilon}\right)(1-\varepsilon)^{p}}{M_{l}^{2} \rho(\theta+l)}
\end{aligned}
$$

holds. Similarly by (3.1c), (3.2b), and (3.1d),

$$
\begin{aligned}
\varepsilon^{p} & >\left\|\omega_{2}\right\|^{p} \geq \frac{\rho\left(\theta+t_{\varepsilon}\right)\left\|c_{\varepsilon} T\left(t_{\varepsilon}\right) \omega_{2}\right\|^{p}}{M_{l}^{2}\left|c_{\varepsilon}\right|^{p} \rho(\theta+l)} \\
& >\frac{\rho\left(\theta+t_{\varepsilon}\right)(1-\varepsilon)^{p}}{M_{l}^{2}\left|c_{\varepsilon}\right|^{p} \rho(\theta+l)}
\end{aligned}
$$

holds. By (3.2c) and (3.2d), we can verify that $\varepsilon^{2 p}>\left(\frac{(1-\varepsilon)^{p}}{M_{l}^{2} \rho(\theta+l)}\right)^{2} \rho\left(\theta+t_{\varepsilon}\right) \rho\left(\theta-t_{\varepsilon}\right) \geq$ 0 holds. If $\varepsilon$ tends to 0 , then $\rho\left(\theta-t_{\varepsilon}\right) \rho\left(\theta+t_{\varepsilon}\right)$ tends to 0 .

$(\Leftarrow)$ Assume for each $\theta \in \mathbf{R}$ that there exists a sequence $\left\{t_{j}\right\} \subset \mathbf{R}_{+}$such that $\lim _{j \rightarrow \infty} \rho\left(t_{j}+\theta\right) \rho\left(-t_{j}+\theta\right)=0$. Let $y$ and $z$ be any nonzero functions with compact support $[\theta-l, \theta](l>0)$. For $l>0$, there exists $M_{l}$ satisfying (II) by Lemma B. By the assumption, for any $\varepsilon>0$, there exists $t_{j}>l$ such that

$$
\rho\left(t_{j}+\theta\right) \rho\left(-t_{j}+\theta\right)<\frac{(\rho(\theta-l) \varepsilon)^{2}}{M_{l}^{4}\|z\|\left\|^{p}|| y\right\|^{p}}
$$


holds. Put

$$
v_{j}(\tau)= \begin{cases}y(\tau) & \tau \in[\theta-l, \theta], \\ \frac{1}{c_{j}} \cdot z\left(\tau-t_{j}\right) & \tau \in\left[t_{j}+\theta-l, t_{j}+\theta\right], \\ 0 & \text { otherwise }\end{cases}
$$

with $c_{j}=\left(\frac{\|z\|^{p} M_{l}^{2} \rho\left(t_{j}+\theta\right)}{\varepsilon \rho(\theta-l)}\right)^{\frac{1}{p}}$.

By Lemma B and the above inequality, we have

$$
\left\|v_{j}-y\right\|^{p}=\int_{t_{j}+\theta-l}^{t_{j}+\theta}\left|\frac{1}{c_{j}} \cdot z\left(\tau-t_{j}\right)\right|^{p} \rho(\tau) d \tau \leq \frac{1}{c_{j}^{p}} \cdot \frac{M_{l}^{2} \rho\left(t_{j}+\theta\right)}{\rho(\theta-l)}\|z\|^{p}=\varepsilon
$$

and

$$
\left\|c_{j} T\left(t_{j}\right) v_{j}-z\right\|^{p}=\int_{\theta-l-t_{j}}^{\theta-t_{j}}\left|c_{j} \cdot y\left(\tau+t_{j}\right)\right|^{p} \rho(\tau) d \tau \leq c_{j}^{p} \frac{M_{l}^{2} \rho\left(\theta-t_{j}\right)}{\rho(\theta-l)}\|y\|^{p}<\varepsilon .
$$

Therefore $\{T(t)\}$ is supercyclic by Lemma 2 ,

The case $X=C_{0, \rho}(I)$ can be proved in a similar way to the case $L_{\rho}^{p}(I)$.

Remark. It is possible to prove supercyclicity by showing the existence of a special function $x \in X$ such that $\{c T(t) x \mid t \geq 0, c \in \mathbf{R}\}$ is dense in $X$. We shall show that in the case of $X=C_{0, \rho}([0, \infty))$ the translation semigroup on $X$ is supercyclic from the definition directly.

Let $C_{c p t}([0, \infty))$ be the space of continuous functions on $[0, \infty)$ with compact support. Then $C_{c p t}([0, \infty))$ is a dense subset of $X$. Let $C_{c p t, 1}^{0}([0, \infty))$ be the set $\left\{f \in C_{c p t}([0, \infty)) \mid\|f\|_{\infty} \leq 1, f(0)=0\right\}$. Put $s(f)=\sup \{\tau \in[0, \infty) \mid f(\tau) \neq 0\}$ for any $f \in C_{c p t}([0, \infty))$.

Let $F=\left\{f_{k}\right\}_{k=1}^{\infty}$ be a countable subset of $C_{c p t, 1}^{0}([0, \infty])$ such that for any $g \in$ $C_{c p t, 1}^{0}([0, \infty])$ and for any $\varepsilon>0$, there exists $f \in F$ satisfying $\|f-g\|_{\infty}<\varepsilon$ and $|s(f)-s(g)|<1$. Let $F^{\prime}=\left\{f_{1}, f_{2}, f_{1}, f_{3}, f_{2}, f_{1}, f_{4}, f_{3}, f_{2}, f_{1}, \cdots\right\}=\left\{h_{1}, h_{2}, h_{3}, \cdots\right\}$. For $k \in \mathbf{N}$, put $L_{k}=s\left(h_{k}\right)+1, K_{k+1}=\Sigma_{i=1}^{k} L_{i}$ and $\alpha_{k}=\sup _{\tau \in\left[0, K_{k+1}\right]} \rho(\tau)$. Then $\alpha_{k}$ is finite by the definition of an admissible weight function $\rho$. Put $K_{1}=0$, $\beta_{1}=\max \left\{\alpha_{1}, 1\right\}$ and

$$
\beta_{k}=\max \left\{k \alpha_{k}, k \beta_{1} \alpha_{k}, \cdots, k \beta_{k-1} \alpha_{k}\right\}
$$

for $k \geq 2$. Put

$$
x(\tau)= \begin{cases}\frac{1}{\beta_{1}} h_{1}(\tau), & K_{1} \leq \tau<K_{2}, \\ \frac{1}{\beta_{2}} h_{2}\left(\tau-K_{2}\right), & K_{2} \leq \tau<K_{3}, \\ \cdots \cdots & \\ \frac{1}{\beta_{k}} h_{k}\left(\tau-K_{k}\right), & K_{k} \leq \tau<K_{k+1}, \\ \cdots \cdots & \\ \cdots \cdots & \end{cases}
$$

Then $x$ is continuous on $[0, \infty)$, since $h_{j} \in C_{c p t, 1}^{0}([0, \infty))$. So $x$ belongs to $X$ by the following relation:

$$
\begin{aligned}
\lim _{\tau \rightarrow \infty}|x(\tau) \rho(\tau)| & \leq \lim _{k \rightarrow \infty} \sup _{\tau \in\left[K_{k}, K_{k+1}\right]} \frac{1}{\beta_{k}}\left|h_{k}\left(\tau-K_{k}\right)\right| \rho(\tau) \\
& \leq \lim _{k \rightarrow \infty} \frac{1}{\beta_{k}} \cdot \alpha_{k} \leq \lim _{k \rightarrow \infty} \frac{1}{k}=0 .
\end{aligned}
$$

We shall show that for any $f \in X$ and any $\varepsilon>0$, there exist $c \in \mathbf{R}$ and $t \geq 0$ such that $\|f-c T(t) x\|<\varepsilon$. 
Since $C_{c p t}([0, \infty))$ is dense in $X$, there exists $f_{0} \in C_{c p t}([0, \infty))\left(\left\|f_{0}\right\|_{\infty} \neq 0\right)$ such that $\sup _{\tau \in[0, \infty)}\left|\left(f(\tau)-f_{0}(\tau)\right) \rho(\tau)\right|<\frac{\varepsilon}{2}$. Put $K=\sup _{\tau \in\left[0, s\left(f_{0}\right)+2\right]} \rho(\tau)$. There exists $h \in F \operatorname{such}$ that $\sup _{\tau \in[0, \infty)}\left|\frac{f_{0}(\tau)}{|| f_{0} \|_{\infty}}-h(\tau+1)\right|<\frac{\varepsilon}{2 K|| f_{0} \|_{\infty}}$ and $\left|s\left(f_{0}\right)-(s(h)-1)\right|<1$. By the way of construction of $F^{\prime}$, there exist countable numbers $m(1)<m(2)<$ $\cdots<m(j)<\cdots$ such that $h=h_{m(j)} \in F^{\prime}$. For any $j \in \mathbf{N}$, put $t_{j}=K_{m(j)}+1$ and $c_{j}=\beta_{m(j)}\left\|f_{0}\right\|_{\infty}$. Then for $\tau \in[0, s(h)]$ we have $h_{m(j)}(\tau+1)=\beta_{m(j)} x\left(\tau+t_{j}\right)$. So by using the relations $s(h) \leq s\left(f_{0}\right)+2$ and $s\left(f_{0}\right) \leq s(h)$, we have

$$
\begin{aligned}
& \sup _{\tau \in[0, s(h)]}\left|f_{0}(\tau)-c_{j} T\left(t_{j}\right) x(\tau)\right| \rho(\tau)=\sup _{\tau \in[0, s(h)]}\left\|f_{0}\right\|_{\infty}\left|\frac{f_{0}(\tau)}{\left\|f_{0}\right\|_{\infty}}-h(\tau+1)\right| \rho(\tau) \\
& \quad<\left\|f_{0}\right\|_{\infty} \cdot \frac{\varepsilon}{2 K\left\|f_{0}\right\|_{\infty}} \cdot K<\frac{\varepsilon}{2}
\end{aligned}
$$

and

$$
\begin{aligned}
& \sup _{\tau \in[s(h), \infty)}\left|f_{0}(\tau)-c_{j} T\left(t_{j}\right) x(\tau)\right| \rho(\tau)=\sup _{\tau \in[s(h), \infty)}\left|c_{j} T\left(t_{j}\right) x(\tau)\right| \rho(\tau) \\
& =\sup _{k=1,2,3, \cdots} \sup _{\tau \in\left[K_{m(j)+k}-t_{j}, K_{m(j)+k+1}-t_{j}\right]}\left|\left\|f_{0}\right\|_{\infty} \beta_{m(j)} \frac{h_{m(j)+k}\left(\tau+t_{j}-K_{m(j)+k}\right)}{\beta_{m(j)+k}}\right| \rho(\tau) \\
& \leq \sup _{k=1,2,3, \cdots}\left\|f_{0}\right\|_{\infty} \beta_{m(j)} \cdot \frac{\alpha_{m(j)+k}}{\beta_{m(j)+k}} \leq\left\|f_{0}\right\|_{\infty} \cdot \frac{1}{m(j)} .
\end{aligned}
$$

Since $\lim _{j \rightarrow \infty} m(j)=\infty$, we have

$$
\begin{aligned}
\| f_{0}- & c_{j} T\left(t_{j}\right) x \| \\
& \leq \max \left\{\sup _{\tau \in[0, s(h)]}\left|f_{0}(\tau)-c_{j} T\left(t_{j}\right) x(\tau)\right| \rho(\tau), \sup _{\tau \in[s(h), \infty)}\left|f_{0}(\tau)-c_{j} T\left(t_{j}\right) x(\tau)\right| \rho(\tau)\right\} \\
& <\frac{\varepsilon}{2},
\end{aligned}
$$

for sufficiently large $j$. By the inequality $\left\|f-c_{j} T\left(t_{j}\right) x\right\| \leq\left\|f-f_{0}\right\|+$ $\left\|f_{0}-c_{j} T\left(t_{j}\right) x\right\|<\varepsilon$, we get the conclusion.

\section{Chaotic translation semigroups}

In this section, we give necessary and sufficient conditions for the translation semigroup to be chaotic. The necessary and sufficient condition in Theorem A for the translation semigroup to be hypercyclic depends on whether $I=[0, \infty)$ or $I=(-\infty, \infty)$, but does not depend on whether $X$ is $L_{\rho}^{p}(I)$ or $C_{0, \rho}(I)$. The same applies to the supercyclic semigroup. However, as for the chaotic condition, it depends mainly on whether $X$ is $L_{\rho}^{p}(I)$ or $C_{0, \rho}(I)$ and depends slightly on whether $I=[0, \infty)$ or $I=(-\infty, \infty)$. Even if $\lim _{\tau \rightarrow \infty} \rho(\tau)=0$ holds, the translation semigroup on $L_{\rho}^{p}(I)$ is not necessarily chaotic as shown in Example 1 . In the case of $C_{0, \rho}(I), \lim _{\tau \rightarrow \infty} \rho(\tau)=0$ is a sufficient condition for the semigroup to be chaotic as shown in Theorem 4 but not a necessary condition as shown in Example 2.

For the case of $L_{\rho}^{p}$, we have

Theorem 2. Let $I=(-\infty, \infty)$ (resp. $I=[0, \infty)$ ) and let $X$ be $L_{\rho}^{p}(I)$. Then the translation semigroup $\{T(t)\}$ on $X$ is chaotic if and only if for all $\varepsilon>0$ and for all $l>0$, there exists $P>0$ such that

$$
\sum_{n \in \mathbf{Z} \backslash\{0\}} \rho(l+n P)<\varepsilon \quad\left(\text { resp. } \sum_{n=1}^{\infty} \rho(l+n P)<\varepsilon\right) .
$$


Proof. $(\Rightarrow)$ Suppose $\{T(t)\}$ is chaotic on $L_{\rho}^{p}((-\infty, \infty))$. Take $\varepsilon>0, l>0$ and $z \in X$ such that $\|z\|=1$ and $\operatorname{supp}(z) \subset[l, l+\theta]$ for some $\theta>0$. By Lemma B, for $\theta>0$ there exists $M_{\theta} \geq 1$ satisfying (1). Take positive $\varepsilon^{\prime}$ satisfying the following condition:

$$
\left(\varepsilon^{\prime}\right)^{p}<\min \left\{\frac{\varepsilon}{2^{p} M_{\theta}^{2} \rho(l+\theta)}, \frac{1}{2^{p}}\right\} .
$$

Since $X_{\text {per }}$ is dense in $X$, there exists $v \in X_{\text {per }}$ such that $\|z-v\|<\varepsilon^{\prime}$. For $v \in X_{\text {per }}$, there exists $P>0$ such that $v=T(n P) v$ for all $n \in \mathbf{N}$. So we obtain $\|z-T(n P) v\|<\varepsilon^{\prime}$. By replacing $P$ with $m P$ for sufficiently large $m \in \mathbf{N}$, we can choose $P$ such as $P>\theta$. The following assertion holds for each $n \in \mathbf{Z}$. Let $w_{n}$ be the restriction of $v$ to the interval $[l+n P, l+n P+\theta]$. Then $\operatorname{supp}\left(T(n P) w_{n}\right) \subset[l, l+\theta]$ and $\left\|z-T(n P) w_{n}\right\|<\varepsilon^{\prime}$ hold by relations $\operatorname{supp}(z) \subset[l, l+\theta]$ and $\|z-T(n P) v\|<\varepsilon^{\prime}$. So we have $\|z\|-\left\|T(n P) w_{n}\right\|<\varepsilon^{\prime}$ and $\left\|T(n P) w_{n}\right\|>1-\varepsilon^{\prime}>1-\frac{1}{2}=\frac{1}{2}$ since $\|z\|=1$ holds. Next we calculate $\left\|T(n P) w_{n}\right\|$. We obtain the following inequality by Lemma B:

$$
\begin{aligned}
\left\|T(n P) w_{n}\right\|^{p} & =\int_{l}^{l+\theta} \rho(\tau) \cdot\left|T(n P) w_{n}(\tau)\right|^{p} d \tau \\
& =\int_{l+n P}^{l+n P+\theta} \rho(\tau-n P) \cdot\left|w_{n}(\tau)\right|^{p} d \tau \\
& \leq M_{\theta} \cdot \rho(l+\theta) \int_{l+n P}^{l+n P+\theta}\left|w_{n}(\tau)\right|^{p} d \tau .
\end{aligned}
$$

So we obtain

$$
\int_{l+n P}^{l+n P+\theta}|v(\tau)|^{p} d \tau=\int_{l+n P}^{l+n P+\theta}\left|w_{n}(\tau)\right|^{p} d \tau \geq \frac{1}{2^{p} M_{\theta} \rho(l+\theta)} .
$$

Since $\operatorname{supp}(z) \subset[l, l+\theta]$, we have

$$
\begin{aligned}
\left(\varepsilon^{\prime}\right)^{p} & >\|z-v\|^{p} \geq \sum_{n \in \mathbf{Z} \backslash\{0\}} \int_{l+n P}^{l+n P+\theta} \rho(\tau)|v(\tau)|^{p} d \tau \\
& \geq \sum_{n \in \mathbf{Z} \backslash\{0\}} \frac{1}{M_{\theta}} \rho(l+n P) \int_{l+n P}^{l+n P+\theta}|v(\tau)|^{p} d \tau \\
& \geq 1 \sum_{n \in \mathbf{Z} \backslash\{0\}} \frac{\rho(l+n P)}{2^{p} M_{\theta}^{2} \rho(l+\theta)} .
\end{aligned}
$$

So we obtain $\sum_{n \in \mathbf{Z} \backslash\{0\}} \rho(l+n P)<\left(\varepsilon^{\prime}\right)^{p} \cdot 2^{p} M_{\theta}^{2} \rho(l+\theta)<\varepsilon$.

$(\Leftarrow)$ Since the assumption implies that $\liminf _{\tau \rightarrow \infty} \rho(\tau)=0,\{T(t)\}$ is hypercyclic by Theorem A. So we only have to show the set of periodic points $X_{\text {per }}$ is dense in $X$. Since the set $X_{0,0}$ of all the functions with compact support is dense in $X$, we shall show $X_{p e r}$ is dense in $X_{0,0}$. Take $\varepsilon>0$ and $z \in X_{0,0}$. Then there exists $l>0$ such that $\operatorname{supp}(z) \subset[-l, l]$. From Lemma B, there exists $M_{2 l} \geq 1$ satisfying (11). Take $\varepsilon^{\prime}$ such as $0<\varepsilon^{\prime}<\frac{\rho(-l)}{M_{2 l}^{2}\|z\|} \varepsilon$. From the assumption, there exists 
$P>0$ such that $\sum_{n \in \mathbf{Z} \backslash\{0\}} \rho(l+n P)<\varepsilon^{\prime}$. By replacing $P$ with $m P$ for sufficiently large $m \in \mathbf{N}$, we can assume $P>2 l$. We shall construct $v_{p}$ in the following way: $v_{p}(\tau)=\sum_{n \in \mathbf{Z}} z(\tau-n P)$. Then clearly $T(P) v_{p}=v_{p} ;$ we calculate $\left\|z-v_{p}\right\|$ :

$$
\begin{aligned}
\left\|z-v_{p}\right\|^{p} & =\left\|\sum_{n \in \mathbf{Z} \backslash\{0\}} z(\tau-n P)\right\|^{p} \\
& \leq \sum_{n \in \mathbf{Z} \backslash\{0\}} \int_{-\infty}^{\infty} \rho(\tau) \cdot|z(\tau-n P)|^{p} d \tau \\
& =\sum_{n \in \mathbf{Z} \backslash\{0\}} \int_{-l+n P}^{l+n P} \frac{\rho(\tau)}{\rho(\tau-n P)} \cdot \rho(\tau-n P) \cdot|z(\tau-n P)|^{p} d \tau \\
& \leq \sum_{n \in \mathbf{Z} \backslash\{0\}} \frac{M_{2 l}^{2} \rho(l+n P)}{\rho(-l)} \cdot\|z\|^{p} \\
& =\frac{M_{2 l}^{2}\|z\|^{p}}{\rho(-l)}\left\{\sum_{n \in \mathbf{Z} \backslash\{0\}} \rho(l+n P)\right\} \leq \varepsilon .
\end{aligned}
$$

(In the case of $I=[0, \infty)$, replace $-l, 2 l, \mathbf{Z}$ and $\mathbf{Z} \backslash\{0\}$ with $0, l, \mathbf{Z}_{+}$and $\mathbf{N}$.)

Moreover $v_{p} \in L_{\rho}^{p}(I)$ and $v_{p} \in X_{p e r}$. So $X_{p e r}$ is dense in $X$. Therefore $\{T(t)\}$ is chaotic.

The following example shows that even if $\lim _{\tau \rightarrow \infty} \rho(\tau)=0$ holds, the condition in Theorem 2 is not satisfied.

Example 1. Let

$$
\rho(\tau)= \begin{cases}1 & \text { for } 0 \leq \tau \leq 1, \\ \frac{1}{\tau} & \text { for } 1<\tau .\end{cases}
$$

Then $\rho$ is an admissible function on $[0, \infty)$ and $\lim _{\tau \rightarrow \infty} \rho(\tau)=0$. While for any $P>0$, there exists $m \in \mathbf{N}$ such that $m>\frac{1}{P}$. So $\sum_{n=1}^{\infty} \rho(1+n P)=\sum_{n=1}^{\infty} \frac{1}{1+n P} \geq$ $\sum_{n=1}^{\infty} \frac{1}{P} \frac{1}{m+n}=\infty$. Therefore for $l=1$ and for any $\varepsilon>0$, there does not exist $P>0$ such that $\sum_{n \in \mathbf{Z} \backslash\{0\}} \rho(l+n P)<\varepsilon$.

For the case of $C_{0, \rho}$, we have

Theorem 3. Let $I=(-\infty, \infty)$ (resp. $I=[0, \infty))$ and let $X$ be $C_{0, \rho}(I)$. Then the following assertions are equivalent:

(1) the translation semigroup $\{T(t)\}$ on $X$ is chaotic;

(2) for all $\varepsilon>0$ and for all $l>0$, there exists $P>0$ such that $\rho(l+n P)<\varepsilon$ for all $n \in \mathbf{Z} \backslash\{0\} \quad$ (resp. $n \in \mathbf{N})$;

(3) there exists $\left\{l_{i}\right\}_{i=1}^{\infty} \subset \mathbf{R}^{+}\left(l_{i} \rightarrow \infty\right.$ as $\left.i \rightarrow \infty\right)$ such that for all $\varepsilon>0$ and for all $i \in \mathbf{N}$ there exists $P>0$ such that $\rho\left(l_{i}+n P\right)<\varepsilon$ for all $n \in \mathbf{Z} \backslash\{0\}$ (resp. $n \in \mathbf{N})$.

Proof. (1) implies (2): Suppose $\{T(t)\}$ is chaotic. Take $\varepsilon>0, l>0$ and $z \in X$ with compact support such that $z(l) \neq 0$. Take $\varepsilon^{\prime}$ such as $0<\varepsilon^{\prime}<\min \left\{\frac{|z(l)| \rho(l)}{2}, \frac{z(l)}{2}\right\}$. Since $X_{p e r}$ is dense in $X$, there exists $v \in X_{\text {per }}$ such that $\|z-v\|<\varepsilon^{\prime}$. For $v \in X_{\text {per }}$, there exists $P>0$ such that $v=T(n P) v$ for all $n \in \mathbf{Z}$ (resp. $n \in \mathbf{N}$ ). Then $\varepsilon^{\prime}>\|z-v\| \geq \sup _{\tau \in I} \rho(\tau)(|z(\tau)|-|v(\tau)|) \geq \rho(l)(|z(l)|-|v(l)|)$ holds. So 
we have $|v(l)|>|z(l)|-\frac{\varepsilon^{\prime}}{\rho(l)}$. By replacing $P$ with $m P$ for sufficiently large $m \in \mathbf{N}$, we can choose $P>0$ such that $l \pm n P$ (resp. $l+n P) \notin \operatorname{supp}(z)$ for all $n \in \mathbf{N}$. Then we obtain the following inequalities for each $n \in \mathbf{Z} \backslash\{0\}$ (resp. $n \in \mathbf{N}$ ):

$$
\varepsilon^{\prime}>\|z-v\| \geq \rho(l+n P) \cdot|v(l+n P)|=\rho(l+n P) \cdot|v(l)|>\rho(l+n P) \cdot\left(|z(l)|-\frac{\varepsilon^{\prime}}{\rho(l)}\right) .
$$

So we have $\rho(l+n P)<\varepsilon^{\prime} /\left(|z(l)|-\frac{\varepsilon^{\prime}}{\rho(l)}\right)<\varepsilon$. Therefore for all $l>0$ and for all $\varepsilon>0$, there exists $P>0$ such that $\rho(l+n P)<\varepsilon$ for all $n \in \mathbf{Z} \backslash\{0\}$ (resp. $n \in \mathbf{N})$.

(2) implies (3): It is clear.

(3) implies (2): Take $\varepsilon>0$ and $l>0$. Then there exists $i_{0} \in \mathbf{N}$ such that $l \in\left[l_{i_{0}-1}, l_{i_{0}}\right)$. Let $L$ be $l_{i_{0}}-l$. By Lemma B, there exists $M_{L} \geq 1$ satisfying (11). Take positive $\varepsilon^{\prime}<\frac{\varepsilon}{M_{L}}$. Then from assumption, there exists $P>0$ such that $\rho\left(l_{i_{0}}+n P\right)<\varepsilon^{\prime}$ for all $n \in \mathbf{Z} \backslash\{0\}$ (resp. $n \in \mathbf{N}$ ). So we obtain

$$
\rho(l+n P) \leq M_{L} \rho(l+n P+L)=M_{L} \rho\left(l+n P+l_{i_{0}}-l\right)=M_{L} \rho\left(l_{i_{0}}+n P\right)<\varepsilon .
$$

(2) implies (1): It is clear that $\{T(t)\}$ is hypercyclic by Theorem A. So we only have to show the set $X_{\text {per }}$ of periodic points is dense in $X$. Since the set $X_{0,0}$ of all the functions with compact support is dense in $X$, we shall show $X_{p e r}$ is dense in $X_{0,0}$ and the proof is analogous to that of the case of $L_{\rho}^{p}$ as follows. Take $\varepsilon>0$ and $z \in X_{0,0}$. Then there exists $l>0$ such that $\operatorname{supp}(z) \subset[-l, l]$. From Lemma B, there exists $M_{2 l} \geq 1$ satisfying (11). Take $\varepsilon^{\prime}$ such as $0<\varepsilon^{\prime}<\frac{\rho(-l)}{M_{2 l}^{2}\|z\|} \varepsilon$. Then from assumption, there exists $P>0$ such that $\rho(l+n P)<\varepsilon^{\prime}$ for all $n \in \mathbf{Z} \backslash\{0\}$. By replacing $P$ with $m P$ for sufficiently large $m \in \mathbf{N}$, we can choose $P>0$ such that $2 l<P$. We shall put $v_{p}(\tau)=\sum_{n \in \mathbf{Z}} z(\tau-n P)$. Then clearly $T(P) v_{p}=v_{p}$; we calculate $\left\|z-v_{p}\right\|:$

$$
\begin{aligned}
\left\|z-v_{p}\right\|=\left\|\sum_{n \in \mathbf{Z} \backslash\{0\}} z(\tau-n P)\right\| & \leq \sup _{n \in \mathbf{Z} \backslash\{0\}} \sup _{\tau \in[-l+n P, l+n P]} \rho(\tau) \cdot|z(\tau-n P)| \\
& \leq \sup _{n \in \mathbf{Z} \backslash\{0\}} \frac{M_{2 l}^{2} \rho(l+n P)}{\rho(-l)} \cdot\|z\|<\varepsilon .
\end{aligned}
$$

(In the case of $I=[0, \infty)$, replace $-l, 2 l, \mathbf{Z}$ and $\mathbf{Z} \backslash\{0\}$ with $0, l, \mathbf{Z}_{+}$and $\mathbf{N}$.) Hence $v_{p} \in C_{0, \rho}(I)$ and $v_{p} \in X_{p e r}$, so $X_{p e r}$ is dense in $X$. Therefore $\{T(t)\}$ is chaotic.

Condition (3) in Theorem 3 is a stronger condition than $\liminf _{\tau \rightarrow \infty} \rho(\tau)=0$. But this does not imply that $\lim _{\tau \rightarrow \infty} \rho(\tau)=0$ as shown in the following example.

Example 2. There exists an admissible weight function $\rho$ on $[0, \infty)$ which satisfies condition (3) in Theorem 3 and does not converge to 0 as $\tau \rightarrow \infty$.

Proof. Let $L C M\left\{t_{j}\right\}_{j}$ be the least common multiple of $\left\{t_{j}\right\}_{j}$. We construct the following sequences $\left\{t_{j}\right\}_{j=0}^{\infty}$ and $\left\{c_{j}\right\}_{j=2}^{\infty}$ by induction:

$$
\begin{aligned}
t_{0} & =0, \quad t_{1}=2, \quad t_{2}=6, \\
c_{j} & =L C M\left\{t_{j}-t_{1}, t_{j}-t_{2}, \ldots, t_{j}-t_{j-1}\right\} \text { for } j \geq 2, \\
t_{j} & =t_{j-1}+c_{j-1} \text { for } j \geq 3 .
\end{aligned}
$$

Then $\left\{t_{j}\right\}$ and $\left\{c_{j}\right\}$ are increasing sequences. Put

$$
\rho(x)= \begin{cases}e^{-\left(x-t_{j}\right)} & \text { for } x \in\left(t_{j}, t_{j+1}\right] \\ 0 & x=0\end{cases}
$$


Then $\rho$ is measurable. First we shall show that $\rho(x) \leq e^{t} \rho(x+t)$ for all $x \in[0, \infty)$ and $t>0$. Take $x \in[0, \infty)$ and $t>0$. Then there exists $i \in \mathbf{N}$ such that $x+t \in\left(t_{i}, t_{i+1}\right]$ and $\rho(x+t)=e^{-\left(x+t-t_{i}\right)}$. If $x \in\left(t_{i}, t_{i+1}\right]$, then $\rho(x)=e^{-\left(x-t_{i}\right)}=$ $e^{t} \cdot e^{-\left(x+t-t_{i}\right)}=e^{t} \rho(x+t)$. If $x \leq t_{i}$, then $\rho(x) \leq 1=e^{x+t-t_{i}} \rho(x+t) \leq e^{t} \rho(x+t)$. So $\rho$ is an admissible weight function. Next we shall show that for all $\varepsilon>0$ and for all $i \in \mathbf{N}$ there exists $P>0$ such that $\rho\left(t_{i}+n P\right)<\varepsilon$ for all $n \in \mathbf{N}$. Since $\left\{c_{j}\right\}$ is an increasing sequence, there exists $m_{0} \in \mathbf{N}$ such that $\rho\left(t_{m}\right)=e^{-\left(t_{m}-t_{m-1}\right)}=$ $e^{-c_{m-1}}<\varepsilon$ for $m>m_{0}$. For any $i \in \mathbf{N}$, put $m_{i}=\max \left\{i, m_{0}\right\}+1$ and $P_{i}=$ $t_{m_{i}}-t_{i}$. Then $e^{-P_{i}}<\rho\left(t_{m_{i}}\right)<\varepsilon$. For any $n \in \mathbf{N}$ there exists $m_{n}>m_{0}$ satisfying $t_{m_{n}}+P_{i} \leq t_{i}+n P_{i} \leq t_{m_{n}+1}$. Then $\rho\left(t_{i}+n P_{i}\right) \leq \rho\left(t_{m_{n}}+P_{i}\right)<e^{-P_{i}}<\varepsilon$. Therefore $\rho$ satisfies assertion (3) of Theorem 3 and it is clear that $\lim \sup _{\tau \rightarrow \infty} \rho(\tau)=1$ and $\rho(\tau)$ does not converge to 0 as $\tau \rightarrow \infty$.

The condition that $\lim _{\tau \rightarrow \infty} \rho(\tau)=0$ implies more than the fact that $X_{p e r}$ is dense in $X$ as shown in the following theorem.

Theorem 4. Let $I$ be the interval $(-\infty, \infty)$ (resp. $I=[0, \infty)$ ), and let $X$ be $C_{0, \rho}(I)$. For the translation semigroup $\{T(t)\}$ on $X$, the following assertions are equivalent:

(1) $\lim _{\tau \rightarrow \pm \infty} \rho(\tau)=0$ (resp. $\left.\tau \rightarrow \infty\right)$;

(2) $\{T(t)\}$ is chaotic. In addition, for all $\varepsilon>0$ and for all $x \in X$ there exists $t_{0}>0$ such that for all $t \geq t_{0}$ there exists $v_{t} \in X_{\text {per }}$ satisfying $\left\|x-v_{t}\right\|<\varepsilon$ and $T(t) v_{t}=v_{t}$.

Proof. (1) implies (2): It is clear that $\{T(t)\}$ is chaotic from Theorem 3, Take $\varepsilon>0$ and $x \in X$ with compact support. Then there exists $l>0$ such that $\operatorname{supp}(x) \subset[-l, l]$.

Put $\varepsilon^{\prime}>0$ such as $0<\varepsilon^{\prime}<\frac{\rho(-l)}{M_{2 l}^{2}\|x\|} \varepsilon$. Then from assumption, there exists $P_{0}>2 l$ such that $\rho(l+n P)<\varepsilon^{\prime}$ for all $n \in \mathbf{Z} \backslash\{0\}$ and for all $P>P_{0}$.

For each $P>P_{0}$, if we put $v_{p}$ such as $v_{p}(\tau)=\sum_{n \in \mathbf{Z}} x(\tau-n P)$, then clearly $T(P) v_{p}=v_{p}$ and $v_{p} \in X_{p e r}$.

By the same calculation as in the proof of Theorem 3 (from (2) to (1)), we obtain $\left\|x-v_{p}\right\|<\varepsilon$.

(2) implies (1): We shall prove that for all $\varepsilon>0$ there exists $t_{0}>0$ such that for all $t>t_{0}, \rho( \pm t)<\varepsilon$ (resp. $\rho(t)<\varepsilon$ ). Take $x \in X$ with compact support such that $x(0) \neq 0$ and $\varepsilon^{\prime}$ such as $0<\varepsilon^{\prime}<\min \left\{\frac{|x(0)| \rho(0)}{2}, \frac{x(0)}{2}\right\}$.

By assumption there exists sufficiently large $t_{0}>0$ such that $\left(\left(-\infty,-t_{0}\right) \cup\right.$ $\left.\left(t_{0}, \infty\right)\right) \cap \operatorname{supp}(x)=\emptyset\left(\operatorname{resp} .\left(t_{0}, \infty\right) \cap \operatorname{supp}(x)=\emptyset\right)$ and for all $t>t_{0}$, there exists $v_{t} \in X_{\text {per }}$ such that $\left\|x-v_{t}\right\|<\varepsilon^{\prime}$ and $T(t) v_{t}=v_{t}$. For each $t>t_{0}$, we obtain $\rho( \pm t)<\varepsilon$ (resp. $\rho(t)<\varepsilon$ ) by the same calculation as in the proof of Theorem 3 (from (1) to (2)). Then it indicates $\lim _{\tau \rightarrow \pm \infty} \rho(\tau)=0 \quad\left(\right.$ resp. $\lim _{\tau \rightarrow \infty} \rho(\tau)=$ $0)$.

\section{ACKNOWLEDGEMENT}

The authors would like to thank the referee for pointing out that Lemmas 1 , 2 and a part of Theorem 1 hold for a strongly continuous semigroup instead of a translation semigroup. 


\section{REFERENCES}

1. W. Desch, W. Schappacher and G. F. Webb, Hypercyclic and chaotic semigroups of linear operators, Ergod. Th. \& Dynam. Sys., 17 (1997), 793-819. MR 98j:47083

2. D. A. Herrero, Z-Y. Wang, Compact Perturbations of Hypercyclic and Supercyclic Operators, Indiana Univ. Math. J., 39 (1990), 819-829. MR 91k:47042

3. D. A. Herrero, Limits of Hypercyclic and Supercyclic operators, Journal of Functional Analysis., 99 (1991), 179-190. MR 93e:47001

Doctoral Research Course in Human Culture, Ochanomizu University, 2-1-1 Otsuka, BUNKYO-KU, TOKYO, 112-8610, JAPAN

E-mail address: mai@tke.att.ne.jp

Graduate School of Humanities and Sciences, Ochanomizu University, 2-1-1 Otsuka, BUNKYO-KU, TOKYO, 112-8610, JAPAN

E-mail address: yamada@xx.is.ocha.ac.jp

Department of Information Sciences, Ochanomizu University, 2-1-1 Otsuka, BunkyoKU, TOKYO, 112-8610, JAPAN

E-mail address: takeo@is.ocha.ac.jp 